\title{
How structure sculpts function: Unveiling the contribution of anatomical connectivity
}

to the brain's spontaneous correlation structure

R. G. Bettinardi, G. Deco, V. M. Karlaftis, T. J. Van Hartevelt, H. M. Fernandes, Z. Kourtzi, M. L. Kringelbach, and G. Zamora-López

Citation: Chaos 27, 047409 (2017); doi: 10.1063/1.4980099

View online: http://dx.doi.org/10.1063/1.4980099

View Table of Contents: http://aip.scitation.org/toc/cha/27/4

Published by the American Institute of Physics

\section{Articles you may be interested in}

Structural connectome topology relates to regional BOLD signal dynamics in the mouse brain

Chaos: An Interdisciplinary Journal of Nonlinear Science 27, 047405 (2017); 10.1063/1.4979281

Editorial: On the relation of dynamics and structure in brain networks

Chaos: An Interdisciplinary Journal of Nonlinear Science 27, 047201 (2017); 10.1063/1.4981391

Multilayer motif analysis of brain networks

Chaos: An Interdisciplinary Journal of Nonlinear Science 27, 047404 (2017); 10.1063/1.4979282

Detecting switching and intermittent causalities in time series

Chaos: An Interdisciplinary Journal of Nonlinear Science 27, 047403 (2017); 10.1063/1.4979046

Persistent homology of time-dependent functional networks constructed from coupled time series Chaos: An Interdisciplinary Journal of Nonlinear Science 27, 047410 (2017); 10.1063/1.4978997

Nonlinear resonances and multi-stability in simple neural circuits

Chaos: An Interdisciplinary Journal of Nonlinear Science 27, 013118 (2017); 10.1063/1.4974028 


\title{
How structure sculpts function: Unveiling the contribution of anatomical connectivity to the brain's spontaneous correlation structure
}

\author{
R. G. Bettinardi, ${ }^{1,2, a)}$ G. Deco,,${ }^{1,2,3}$ V. M. Karlaftis, ${ }^{4}$ T. J. Van Hartevelt, ${ }^{5,6}$ \\ H. M. Fernandes, ${ }^{5,6}$ Z. Kourtzi, ${ }^{4}$ M. L. Kringelbach, ${ }^{5,6}$ and G. Zamora-López ${ }^{1,2, b)}$ \\ ${ }^{1}$ Center for Brain and Cognition, Universitat Pompeu Fabra, Barcelona, Spain \\ ${ }^{2}$ Department of Information and Communication Technologies, Universitat Pompeu Fabra, Barcelona, Spain \\ ${ }^{3}$ Institució Catalana de la Recerca i Estudis Avançats, Universitat Pompeu Fabra, Barcelona, Spain \\ ${ }^{4}$ Department of Psychology, University of Cambridge, Cambridge, United Kingdom \\ ${ }^{5}$ Department of Psychiatry, University of Oxford, Oxford, United Kingdom \\ ${ }^{6}$ Center for Music in the Brain, Aarhus University, Aarhus, 8000 Aarhus C, Denmark
}

(Received 1 November 2016; accepted 3 April 2017; published online 17 April 2017)

Intrinsic brain activity is characterized by highly organized co-activations between different regions, forming clustered spatial patterns referred to as resting-state networks. The observed coactivation patterns are sustained by the intricate fabric of millions of interconnected neurons constituting the brain's wiring diagram. However, as for other real networks, the relationship between the connectional structure and the emergent collective dynamics still evades complete understanding. Here, we show that it is possible to estimate the expected pair-wise correlations that a network tends to generate thanks to the underlying path structure. We start from the assumption that in order for two nodes to exhibit correlated activity, they must be exposed to similar input patterns from the entire network. We then acknowledge that information rarely spreads only along a unique route but rather travels along all possible paths. In real networks, the strength of local perturbations tends to decay as they propagate away from the sources, leading to a progressive attenuation of the original information content and, thus, of their influence. Accordingly, we define a novel graph measure, topological similarity, which quantifies the propensity of two nodes to dynamically correlate as a function of the resemblance of the overall influences they are expected to receive due to the underlying structure of the network. Applied to the human brain, we find that the similarity of wholenetwork inputs, estimated from the topology of the anatomical connectome, plays an important role in sculpting the backbone pattern of time-average correlations observed at rest. (C) 2017 Author $(s)$. All article content, except where otherwise noted, is licensed under a Creative Commons Attribution (CC BY) license (http://creativecommons.org/licenses/by/4.0/).

[http://dx.doi.org/10.1063/1.4980099]

The quest to understand how structure shapes function lies at the heart of a broad spectrum of disciplines, ranging from biology to network science. For over a decade, many efforts have been devoted to investigating the impact of different network features, e.g., hubs, clustering, or communities, on the collective behaviour of dynamical processes on complex networks such as spreading phenomena and synchronization. However, a unique answer to this question is not possible, because the emerging network activity is a product of the interplay between the network's topology, the particular local dynamics governing nodes' behavior, and the coupling function defining how information is transferred: network's topology shapes, but does not determine, the collective dynamics. The question is thus whether we can estimate what is the contribution of the structure alone, and which are the most relevant topological features in sculpting the emergent functional relations. Here, we have shown that the global path structure of the network is what truly determines the contribution of the network over the collective dynamics, as it implicitly incorporates information about all other network features,

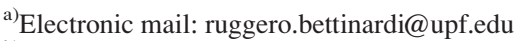

b)Electronic mail: gorka@Zamora-Lopez.xyz
}

e.g., degree-distributions or modules. The expected magnitude of synchrony or correlation between two nodes is largely governed by the common inputs they receive from all other nodes, given that information propagates along all possible paths of any length. We quantify this pairwise, whole-network affinity introducing a network measure, the topological similarity $(\mathcal{T})$. Formally, $\mathcal{T}$ is the direct relation between the structure of a network and the pattern of functional relations that it tends to produce. Applied to the human brain, we find that the similarity of whole-network inputs, defined by the topology of the underlying anatomical connectome, plays an important role in sculpting the backbone pattern of time-average correlations observed at rest. This confirms the pivotal relevance of the path structure in sculpting the network's correlations due to spontaneous activity.

\section{INTRODUCTION}

The quest to understand how structure shapes function lies at the heart of a broad spectrum of disciplines, ranging from molecular biology to network science. A classic example 
in biology is to predict the functional relevance of proteins' three-dimensional structure, ${ }^{1,2}$ whereas in network science, the challenge is to isolate those graph features which drive the interaction patterns arising when a complex network hosts a dynamical process, such as synchronization ${ }^{3,4}$ and spreading phenomena. ${ }^{5,6}$ However, it might not be possible to explain emergent network behavior just as a function of its underlying architecture, as this results from the inextricable interplay between structural and dynamical factors, e.g., those controlling intrinsic properties of the nodes. ${ }^{7-10}$

The relationship between the structure and the function is particularly relevant in neuroscience, as it has been repeatedly shown that morphological and structural variations of the nervous system tend to be associated with behavioral changes due to alterations of intrinsic brain activity's organization. ${ }^{11-13}$ In the last two decades, a large body of research has demonstrated that spontaneous brain fluctuations form structured patterns of consistent co-activations across different subsets of regions. ${ }^{14-18}$ Many efforts have been devoted to reproducing resting-state brain activity by means of computational modeling. Early models based on the structural connectomes of cats and macaques explored the emerging patterns of correlations in those networks at different spatial and temporal scales. ${ }^{19-21}$ With the arrival of structural human connectomes obtained via diffusion imaging and tractography, computational models could be validated with the empirical correlation structure observed in the human resting-state, referred to as functional connectivity (FC) ${ }^{22-26}$ Systematic comparisons showed that using different models to simulate the activity of brain regions returns correlation matrices of varying accuracy to fit the empirical ones. ${ }^{10,27}$ So far, we still lack a unitary model of the relationship between the shape of the brain's connectome and the emergent activity patterns. One of the main reasons is that interactions between different areas do not only depend on the structure of the connectome but also on the local and global dynamics characterizing a given brain state, such as rest, sleep, and anesthesia. ${ }^{28-30}$ The existence of different statedependent activity patterns sustained by the same underlying anatomy exposes how elusive is the relationship between the network structure and function.

In the present paper, we aim at unveiling what is the expected contribution of network topology on the pattern of interactions it naturally tends to generate and sustain. To this aim, in Section II, we summarize the main topological features that contribute to the routing of information through the network and introduce a novel graph theoretical quantity, $\mathcal{T}$, measuring the similarity of the entire input profiles that two nodes receive from the whole network. This measure, that we named topological similarity, is a generalization of the concept of matching index that explicitly accounts for the fact that, in networks, information travels along all possible paths, not only along the shortest ones, and that its content tends to decay as it moves away from the source. ${ }^{31-34}$ This measure, based on a network's topology, represents the expected time-average correlation structure of the network due to topological constraints.

In Section III, we systematically investigate the contribution of three fundamental topological features, namely, the weight of the links, the length of the path, and the presence of redundant alternative paths. Together, they specify the graph's path structure, which determines how influence spreads through the network and, as a consequence, sculpts the inputs that nodes receive.

Finally, in Section IV, we investigate the contribution of the anatomical connectivity of the human brain to its spontaneous correlation structure. For this, we have calculated our topological similarity measure out of the empirically obtained structural connectome, and we have considered it as a zeroth-order approximation of the cross-correlation matrix that one could expect due to topological constraints. We find that the topological similarity captures a considerable portion of the empirical functional connectivity measured with resting-state fMRI. Then, we ran simulations of the brain's activity by modeling the local region dynamics using the Hopf normal model. The numerically simulated functional connectivity resembles the empirical one more accurately than the topological similarity does. These results corroborate that the anatomical connectivity shapes but does not fully determine the empirical functional connectivity, as the underlying topology, defined by the complex fabric of brain axonal pathways, is only one of the factors leading to the observed collective dynamics.

\section{HOW TOPOLOGY SCULPTS NETWORK INTERACTIONS}

When addressing the relationship between the structure and the function in complex networks, we need to remember that the collective behavior of a set of coupled dynamical units depends on three principal ingredients: (i) the structure of the network, (ii) the local dynamics of the nodes, and (iii) the coupling function determining how information is passed from one node to another. In fact, for a fixed network, changing the local dynamical model of the nodes and the coupling function usually leads to different collective dynamics. ${ }^{8-10}$ Therefore, in order to estimate the contribution of the structure alone we need to isolate, as much as possible, its contribution from that of the other two factors.

Typically, the activity of two nodes exhibits a statistical dependence either if they are connected by means of a direct link or if the aggregate input they receive from the entire network is similar, independent of whether there is a link between them or not. Because information in a network rarely travels exclusively along the shortest paths ${ }^{35,36}$ but instead diffuses along the whole network, the total influence of one node over another due to topological constraints mainly depends on three features: $(a)$ the strength of the coupling between them, usually represented by the weights of the links, $(b)$ the graph distance between the two nodes, and (c) the presence of multiple, alternative, and re-entrant paths between the nodes through which information can travel. ${ }^{37-39}$ We refer to these three features as the topological primitives because, in combination, they characterize the path structure of the network.

In general, the influence of a direct link is greater than the influence exerted over longer indirect paths. In real systems, the "power" of the signals or their information content 
naturally decays along the path, ${ }^{31-34}$ unless there exists an active mechanism which amplifies the signal at the cost of energy. In addition, it is unlikely that the influence or information propagates only along a single, selected path, unless specific gating mechanisms exist to control the routing of information among all existing paths. The total number of paths (non-Hamiltonian walks) of length $l$ between two nodes grows with $l$. This number is given exactly by the $l$ th power of the adjacency matrix $A$, see Ref. 40 . The total number of paths leaving from node $i$ and arriving at node $j$ is given by the sum

$$
\sum_{l=0}^{\infty}\left(A^{l}\right)_{i j}=1+A_{i j}+\left(A^{2}\right)_{i j}+\left(A^{3}\right)_{i j}+\cdots
$$

This number typically diverges and thus, for the dynamics within a network to remain bounded, the amount of influence needs to decay faster with the length than the growth in the number of paths. Mathematically, the problem consists in finding a set of coefficients $\left\{\beta_{l}\right\}$ for which the series $\sum_{l=0}^{\infty} \beta_{l} A^{l}$ converges. A solution to this problem is the communicability $\mathcal{C}$ proposed by Estrada and Hatano. ${ }^{41}$ This measure corresponds to the matrix exponential of $A$, which can be expanded into a series of powers with coefficients $\beta_{l}=1 / l !$

$$
\mathcal{C} \equiv e^{A}=\sum_{l=0}^{\infty} \frac{A^{l}}{l !}=1+A+\frac{A^{2}}{2 !}+\frac{A^{3}}{3 !}+\cdots
$$

From a physical perspective, the communicability is analogous to the Green's function of the network ${ }^{41,42}$ and expresses how local perturbations propagate along the system. Communicability can be tuned using a constant global parameter $g$, that uniformly scales the weights of all links in $A$, allowing to search over multiple scales ${ }^{42,43}$

$$
\mathcal{C}=e^{g A}=\sum_{l=0}^{\infty} \frac{g^{l} A^{l}}{l !}=1+g A+\frac{g^{2} A^{2}}{2 !}+\frac{g^{3} A^{3}}{3 !}+\cdots
$$

When $g$ is weak, perturbations quickly decay, producing local correlations only around the node's neighborhood. As $g$ grows, perturbations propagate deeper into the network, giving rise to stronger correlations over more distant nodes.

The goal of the present work was to address whether it is possible to use information of the topological properties of a network to estimate the most likely correlation structure it tends to exhibit. As argued before, the statistical dependence of two nodes relies not only on the presence of a direct link between them but also on the similarity of the common inputs they are exposed to. We noted that each column vector $\mathbf{c}_{\mathbf{j}}$ of the communicability matrix $\mathcal{C}$ represents the input profile of the influences a node receives from all other nodes along all possible paths. This includes the influence a node exerts on itself through recurrent (or re-entrant) paths. Therefore, if the network hosts a dynamical process, the similarity of the input profiles $\mathbf{c}_{\mathbf{i}}$ and $\mathbf{c}_{\mathbf{j}}$ of nodes $i$ and $j$ could be regarded as an estimate of their expected interdependence. Consequently, we define the topological similarity, $\mathcal{T}_{i j}$, as the cosine similarity between the input profiles of a pair of nodes

$$
\mathcal{T}_{i j}=\frac{\left\langle\mathbf{c}_{\mathbf{i}}, \mathbf{c}_{\mathbf{j}}\right\rangle}{\left\|\mathbf{c}_{\mathbf{i}}\right\|\left\|\mathbf{c}_{\mathbf{j}}\right\|}
$$

where $\langle$,$\rangle is the scalar product and \|\cdot\|$ the vector norm. The definition of $\mathcal{T}$ depends uniquely on the topological constraints of the network encoded in the adjacency matrix $A$, together with the realistic assumption (embedded in $\mathcal{C}$ ) that the influence or the information content decays with the length of the path. Additionally, we find that $\mathcal{T}$ is equivalent to the correlation matrix $R$ of a coupled Gaussian noise diffusion process in which the matrix exponential is considered as the kernel of noise propagation, ${ }^{43}$ see supplementary material.

The topological similarity can be estimated for both directed and undirected graphs, as well as for weighted networks. In the case of undirected graphs, $\mathcal{C}$ is symmetric, but if the links are directed, then $\mathcal{C}$ is asymmetric and its columns determine the input profiles of the nodes while its rows represent the profile of output influences. Despite the measure can be computed for any weighted adjacency matrix, it does not always make sense to do so. Because $\mathcal{T}$ is based on measuring the influence spreading along different paths, it only has a physical meaning when the weights of the links quantify their potential flow capacity or coupling strength. On the other hand, it lacks a physical interpretation when link weights represent other quantities or statistical associations. For example, while it is common in the literature to apply graph measures to binarized functional connectivity matrices, $\mathcal{T}$ shall not be calculated in such cases.

In Section III, we systematically investigate how three network features (the weights of the links, the path length, and the redundancy of paths) mould both the communicability and, as a consequence, the topological similarity between nodes.

\section{ALTERING THE PATH STRUCTURE}

We now focus on investigating how different topological features of the network modulate the influence that a node exerts over another. To this aim, we will focus on three simple classes of graphs: chains, cycles, and path-redundant motifs, see examples in Fig. 1. We will explore how manipulating crucial parameters of these simple graphs leads to changes in the influence between selected pairs of nodes (measured by their communicability, $\mathcal{C}_{i j}$ ) and in their topological similarity $\mathcal{T}_{i j}$. In the case of single link motifs, the contribution of the link's weight is trivial: the weight modulates the mutual influence between the two nodes (see supplementary material Figure S1). The role of the path length is best understood studying simple chain topologies of varying sizes. Increasing the length of the path separating two nodes leads to a decrease in both their communicability and topological similarity, Fig. 1(a). This behavior is directly determined by the decay embedded in the definition of the communicability. From the example, it is indeed evident 

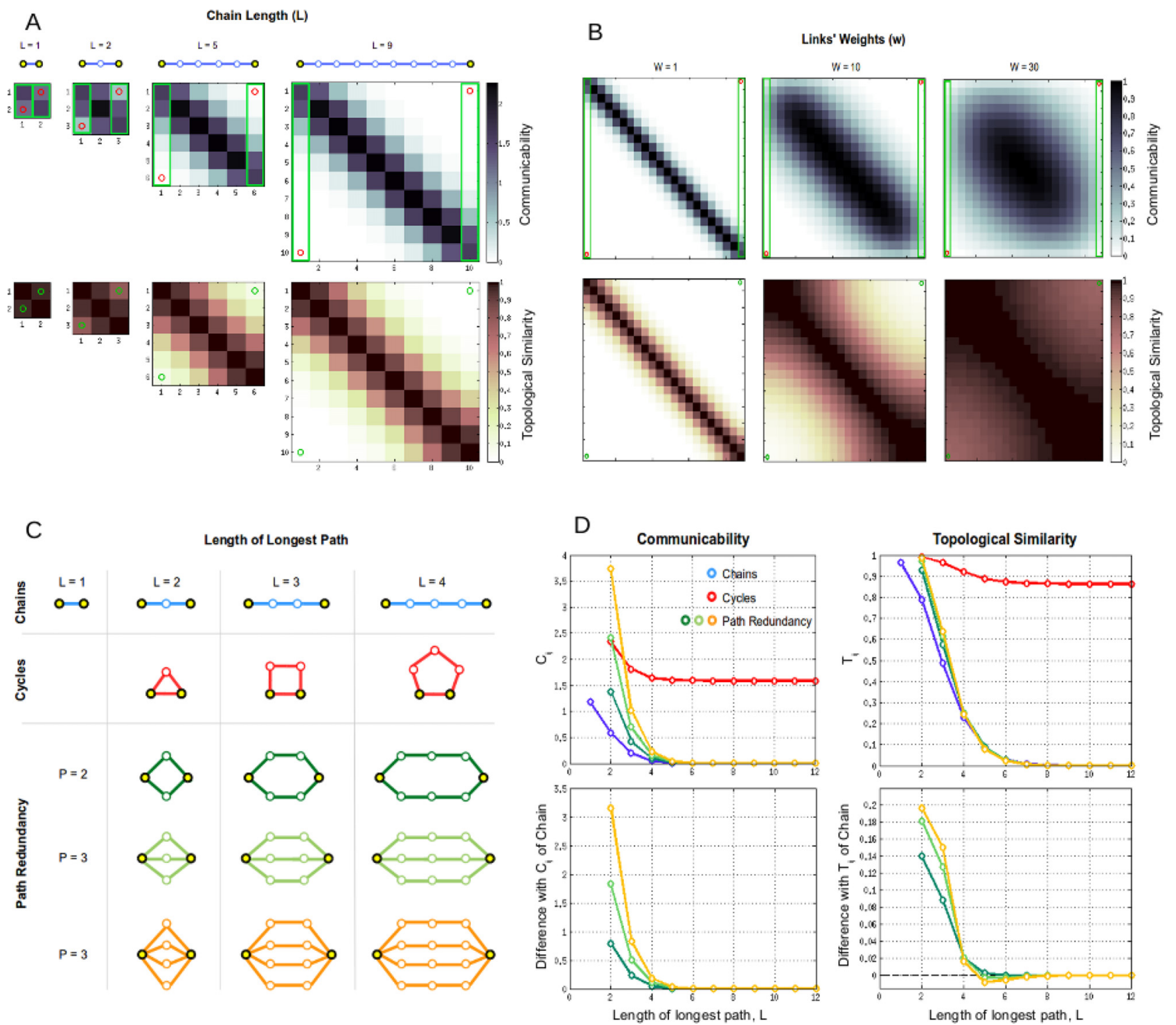

FIG. 1. Behavior of Communicability and Topological Similarity in simple network motifs. (a) Communicability and Topological Similarity matrices of chains of different lengths. In the upper matrices, the red dots indicate the matrix entry corresponding to the communicability between the nodes at the two ends of the chains, whereas the green rectangles mark their whole-network input profiles (column vectors), which are used to calculate the topological similarity of the corresponding nodes (marked with the green dots in the lower matrices. (b) Communicability and Topological Similarity matrices of chains of constant length $(L=21)$ for different links' weights, $w$. See (a) for the legend of red dots, green rectangles, and green dots. (c) Schematic representation of different graphs (chains, cycles, and path-redundant architectures) having a comparable longest path. The reference nodes for which both the communicability $\left(\mathcal{C}_{i j}\right)$ and the topological similarity $\left(\mathcal{T}_{i j}\right)$ where calculated are highlighted in yellow. (d) Upper panels: comparison of $\mathcal{C}_{i j}$ and $\mathcal{T}_{i j}$ of the three different graphs having a comparable longest path. Line colors correspond to those in the schematic representation in (c) (Light blue lines: chains; red lines: cycles; dark green lines: two redundant paths; light green lines: three redundant paths; orange lines: four redundant paths). Lower panels: difference between path-redundant motifs and chains of the same length on the resulting $\mathcal{C}_{i j}$ and $\mathcal{T}_{i j}$. All results were obtained for constant links' weights and global coupling $w=g=1$.

that, for increasing lengths of the chain, the input profiles of the two end nodes become more and more antithetic, due to opposed whole-network influences (see the input profiles highlighted by green rectangles on the communicability matrices, Fig. 1(a)). As a consequence, their topological similarity decreases with path length as well. A uniform variation of the weights of all links in the chain, which corresponds to multiply the links by a constant factor, has mainly a quantitative effect on the decay: increasing the links' weights enhances how far and strong the influence of nodes can travel, Fig. 1(b). The interaction between the chain length and links' weight is shown in supplementary material Figure S2.
Chains can be thought as a baseline to compare more complex motifs. In fact, cycles and path-redundant topologies are built upon chain motifs. We now compare $\mathcal{C}_{i j}$ and $\mathcal{T}_{i j}$ of the three model graphs (chains, cycles, and path-redundant motifs) having an identical diameter, i.e., length of the longest path. Figure 1(c) provides a schematic representation of different motifs of the same longest paths. For the case of chains and path-redundant topologies, we computed $\mathcal{C}_{i j}$ and $\mathcal{T}_{i j}$ for the two nodes at the extremes. For cyclic topologies, we selected two adjacent nodes. This choice allowed us to disentangle the contribution of the indirect paths above and beyond the modulation produced by the direct links. 
In chains, both $\mathcal{C}_{i j}$ and $\mathcal{T}_{i j}$ decay with distance, blue lines in Figure 1(d). On the other hand, the effect of the direct link is well illustrated in the case of cyclic architectures (bottomright panels in Figure 1, red lines). The presence of a direct link importantly enhances $\mathcal{C}_{i j}$ and $\mathcal{T}_{i j}$ and poses a lower bound for them while the contribution due to the indirect path decreases with its length.

The effect of path redundancy is best understood when analyzing the difference between path-redundant motifs and chains of the same length. The two lower panels in Figure 1(d) illustrate three examples of these differences, namely, the cases with two (dark green lines), three (light green lines), and four (orange lines) redundant paths. From this analysis, it becomes evident that increasing the number of alternative paths does enhance both the total influence and the topological similarity between the end nodes and that the magnitude of this increase decays with the length of the paths.

The results of this section show how the weight of the links, the path length, and the presence of alternative routes shape the manner in which the influences of nodes unfold through the graph. These in turn define the most likely pattern of interactions that a network is expected to sustain due to its path structure.

\section{ANATOMICAL ROOTS TO FUNCTIONAL CONNECTIVITY}

In Section III, we have analyzed how very simple topological features shape the interaction between nodes. Real networks, however, are made of intertwined assemblies of those features, forming intricate architectures. As an example of a real complex network, we now study how the anatomical connectivity sculpts the complex pattern of correlations observed in spontaneous brain activity. To this aim, we calculate the topological similarity matrix, $\mathcal{T}_{S C}$, out of the group-average structural connectivity (SC) and consider it as the zero-order approximation of the correlations due to the anatomical path structure. We then compare $\mathcal{T}_{S C}$ to the empirically observed resting-state functional connectivity (FC). The SC matrix is a representation of the brain's wiring diagram, where axonal pathways are reconstructed through diffusion tensor imaging (DTI) and tractography. Despite the reproducibility of current tractography methods, their accuracy to detect crossing fibers and long interhemispheric axons is known to be limited. ${ }^{44-46}$ For these reasons, we perform the analyses twice: for a single hemisphere, Fig. 3(a), and for the whole brain, Fig. 3(b). The details of the experimental procedures are described in Sec. VI.

Adding a uniform coupling strength factor $g$ to the weights of the links allows us to scale how deep do influences propagate into the network (see Sec. II). When $g$ is weak, perturbations quickly decay producing local correlations only around the node's neighborhood. As $g$ grows perturbations propagate deeper into the network, giving rise to stronger correlations over more distant nodes. Figure 2 illustrates three instances of $\mathcal{T}_{S C}$ obtained for $g=0.35,1$ and 2 using the group-average SC matrix of the left hemisphere. For the SCs corresponding to the whole brain and to the left hemisphere, we have scanned through a broad range of global coupling values $g$ to find those returning the $\mathcal{T}_{S C}$ that best approximates the empirical FC, Fig. 3. We quantify the approximation between $\mathcal{T}_{S C}$ and the empirical FC by means of the mean absolute error (MAE), an outlier-robust alternative of the mean squared error, a classic statistic for the goodness of an estimator. Compared to the similarity between the raw SCs and the empirical FCs (dotted lines in panels $\mathrm{G}$ and $\mathrm{H}$ of Fig. 3), $\mathcal{T}_{S C}$ approximates the empirical FC much better, passing from $E(S C, F C)=0.42$ to $E(\mathcal{T}, F C)$ $=0.15$ for the calculations in the left-hemisphere. For the whole brain network, it improves from $E(S C, F C)=0.44$ to $E(\mathcal{T}, F C)=0.22$. The reason behind this improvement is that, as mentioned earlier, the correlation between two nodes is determined not only by the existence of a direct link between them but also by all the common inputs they receive, which depends on all the possible routes through which information can travel. By definition, $\mathcal{T}_{S C}$ accounts for the effect of collateral influences traveling also along indirect paths, while SC only represents direct links between nodes. Moreover, the link weights associated with the SC tracts do not necessarily "predict" the magnitude of the empirical correlation between a pair of brain regions (see supplementary material, Fig. S4).

\section{A. Adding local dynamics}

We now introduce local dynamics to the brain regions and simulate their activity by means of the Hopf normal
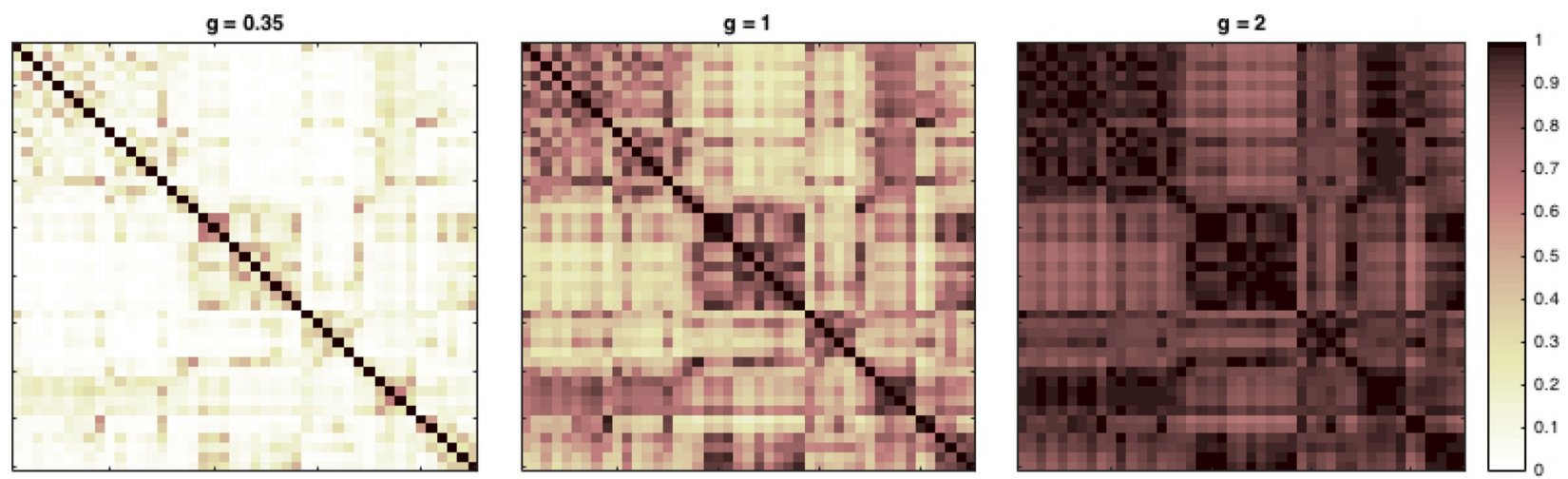

FIG. 2. Effect of the global coupling. Effect of varying the global coupling $g$ onto the resulting topological similarity matrix, $\mathcal{T}$. The three matrices have been obtained from the same empirical structural connectivity matrix (left hemisphere), for $g=0.35,1$ and 2. 

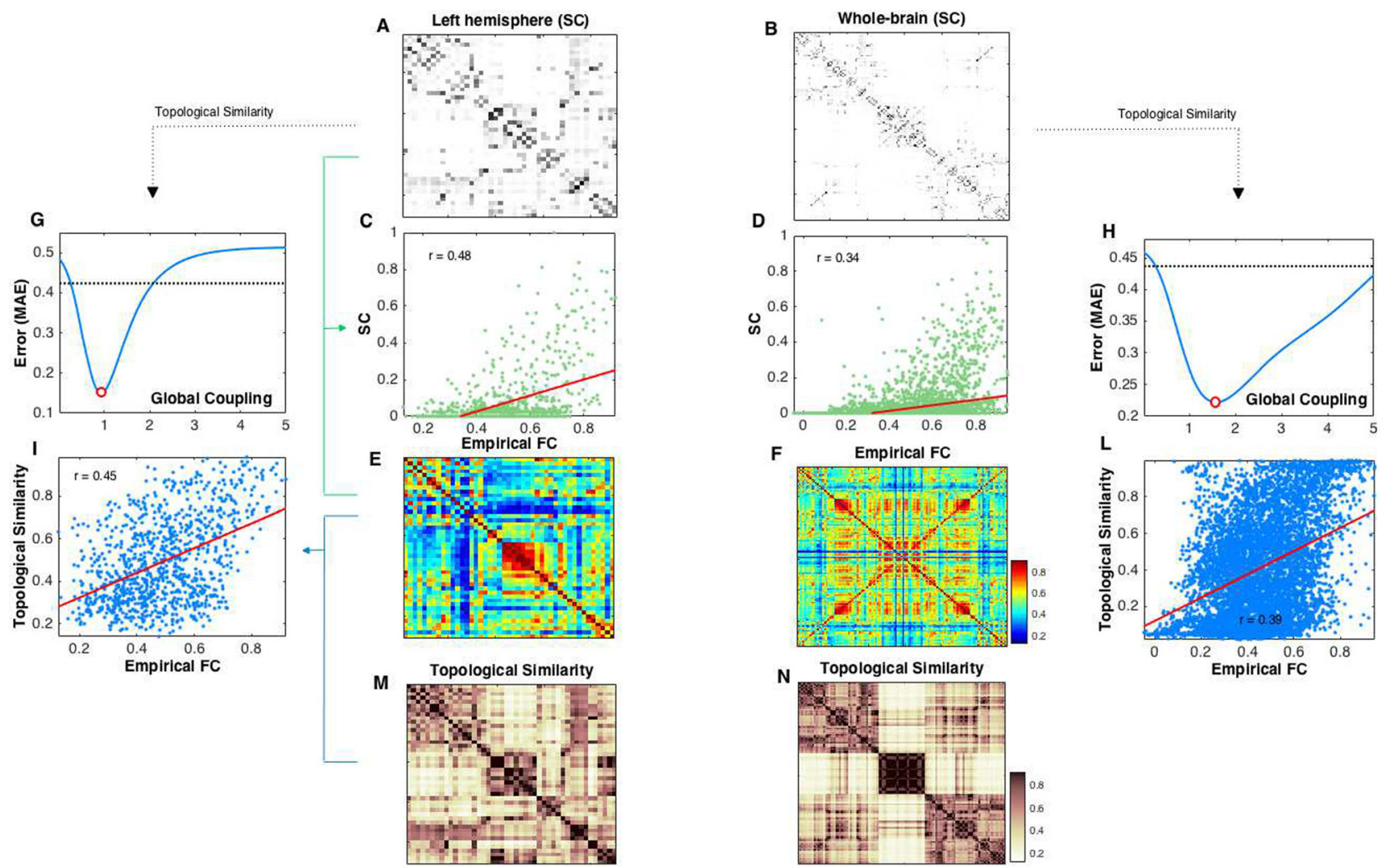

FIG. 3. Contribution of whole-network common inputs to the brain's spontaneous correlation structure. The figure shows results obtained separately for only one hemisphere (Left panels) and for the whole brain (Right panels). (a), (b) structural connectivity matrices (SC). (c), (d) Scatterplots depicting the relationship between SC and the empirical functional connectivity (FC). (e), (f) Empirical functional connectivity matrices (FC). (g), (h) Mean absolute error (MAE) between the empirical FC and the topological similarity $\mathcal{T}$ computed for different values of the global coupling parameter, $g$. The dotted lines correspond to the mean absolute error between the raw SC matrix and the empirical FC. (i), (1) Scatterplots of the empirical FC and the best-fitting topological similarity. (m), (n) Best-fitting Topological similarity matrices. We included also the Pearson's correlation values $r$ corresponding to the best-fitting matrices obtained optimizing the mean absolute error.

model. ${ }^{47,48}$ We then compare the numerically simulated functional connectivity of the whole network with the empirical one. The Hopf model relies on the choice of a single parameter, $\alpha$, controlling the dynamical working-point of each node, see Sec. VI for details. We set $\alpha=0$, meaning that all nodes lie at the edge of the bifurcation, a regime that has been demonstrated to give a good approximation of the empirical resting-state FC and captures the properties of its temporal fluctuations as well. ${ }^{49}$ As done for the topological similarity, we scanned for a range of global coupling values to find the $g$ for which the simulated FC best approximated the empirical FC, panels (A) and (B) in Fig. 4. The introduction of local dynamics gives rise to a correlation structure that is, as expected, closer to the empirical FC than the approximation using $\mathcal{T}$ : indeed, the mean absolute error falls to $E=0.11$ in the case of the left hemisphere and to 0.13 for the whole brain network. The magnitude of the improvement is however small. Together with the strong relationship between the best-fitting simulated FC and $\mathcal{T}$ (see Figure 5), it suggests that most of the similarities between the empirical and the simulated FCs appear to be substantially shaped by the underlying network architecture.

These results demonstrate that knowledge of the topology of whole-network input patterns of different brain regions, sustained by direct and indirect routes of multiple interweaved axonal bundles, can be used to approximate the time-average correlation structure observed from spontaneous BOLD fluctuations beyond the information about direct anatomical connections stored in the SC matrices.

\section{SUMMARY AND DISCUSSION}

In the present paper, we have studied the contribution of topology in shaping the emergent pattern of interactions a network tends to generate. In particular, our goal was to identify the fundamental features behind that contribution. We have shown how three primitive motifs (the strength of the links, the length of the path, and the number of redundant paths) regulate the expected interaction between two nodes, as they alter the overall path structure of the network. A strong direct link is usually a reliable indicator of the magnitude of their interaction. However, the presence of common inputs or redundant paths between them may enhance their interaction beyond the baseline determined by the direct link. When there is no direct link between the nodes, common inputs and redundant paths can still trigger strong correlations between them, although this tends to decrease with the length of the paths. These results show that what truly matters to describe topology's influence on the network dynamics is the global path structure of the network and the weights assigned to each alternative path, since it is unlikely to assume that information would propagate only through shortest paths. Many efforts have been 

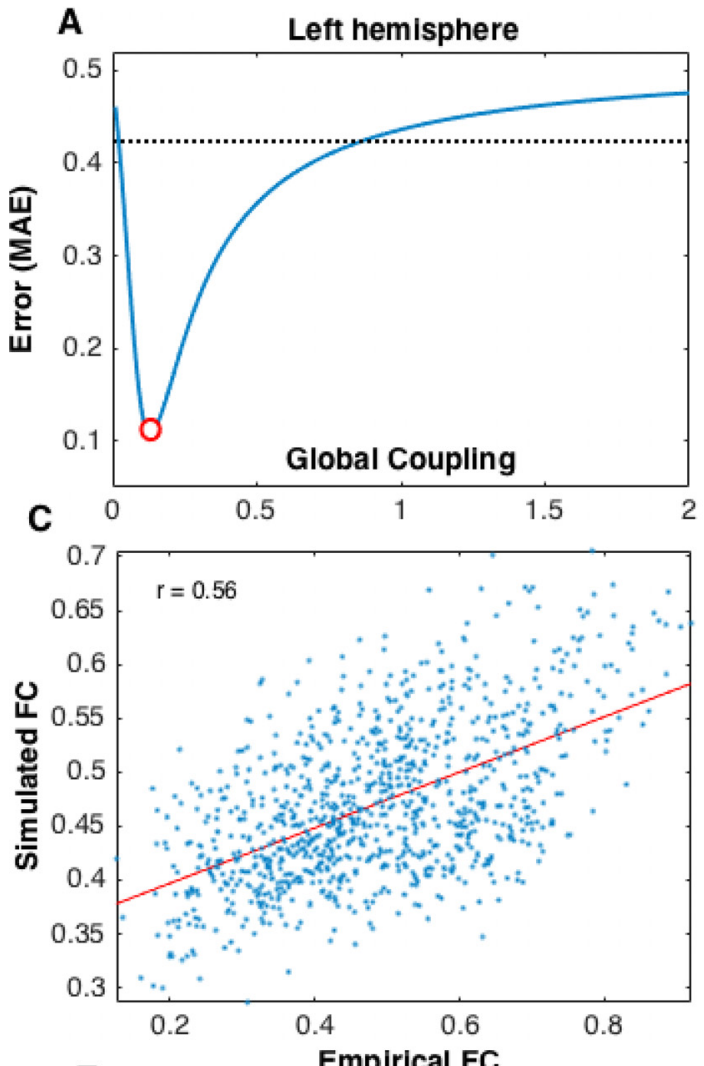

E

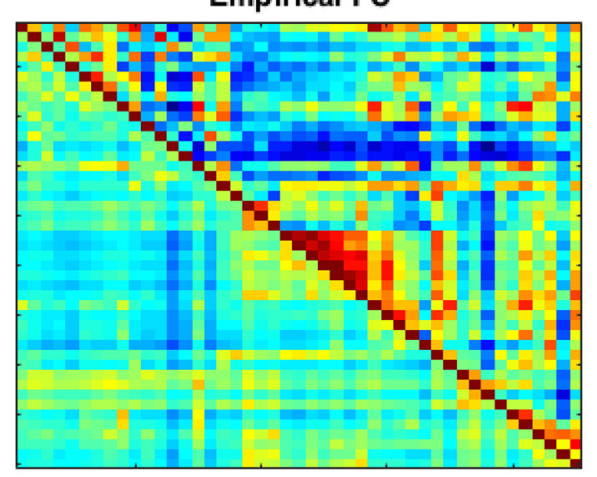

Simulated FC
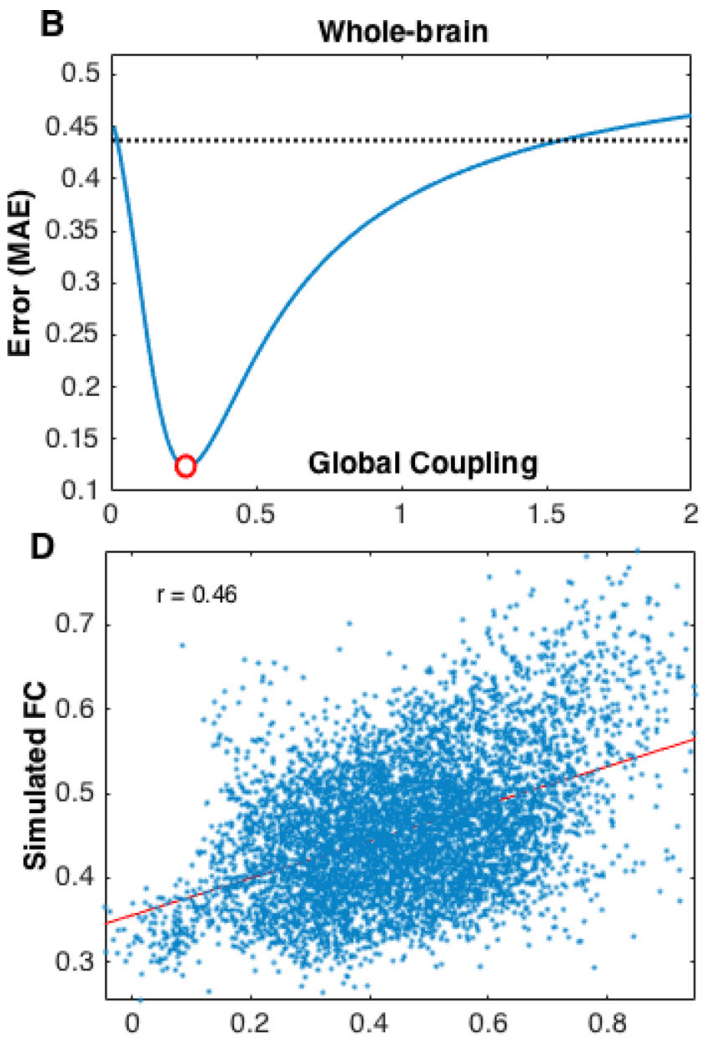

$\mathbf{F}$

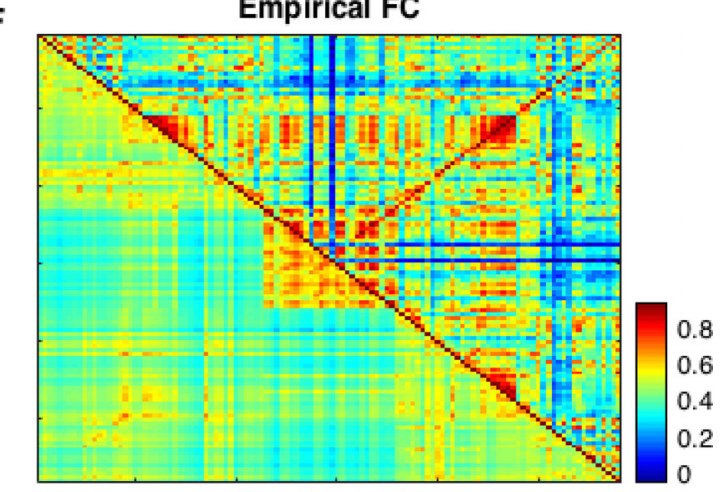

Simulated FC

FIG. 4. Numerical simulations. The figure illustrates the results obtained from numerical simulations using the Hopf normal model. (a), (b) As for data described in Section IV, the quality of the approximation was measured using the mean absolute error (MAE). MAE between empirical structural (SC) and functional connectivity (FC) matrices is indicated by the dotted lines $(\approx 0.42$ in both hemispheres). (c), (d) scatter plots of the empirical functional connectivity (FC) versus the simulated one obtained at the best-fitting global coupling. (e), (f) In both panels, the upper triangles store the empirical FC values, whereas the lower triangles the corresponding ones obtained from simulations at the best fitting value of $g$.
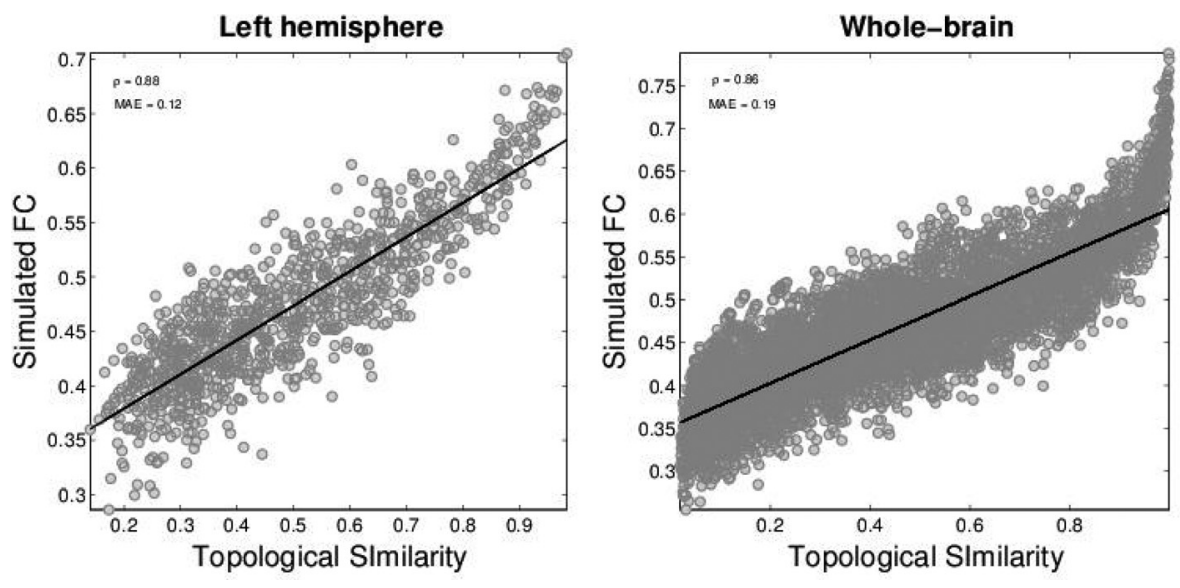

FIG. 5. Relationship between topological similarity and simulated FC. The two panels show the strong relationship between the topological similarity $\mathcal{T}$ and the Hopf-simulated FC (all nodes having $\alpha=0$ ), both separately optimized for the global coupling factor $g$. MAE between $\mathcal{T}$ and the Hopfsimulated FC obtained for one hemisphere (Left panel) is MAE $=0.12$ and the Pearson's correlation between them is $r=0.88$; MAE between $\mathcal{T}$ and the Hopf-simulated FC obtained for the whole-brain (Right panel) is MAE $=0.19$, and the Pearson's correlation between them is $r=0.86$. 
devoted in the past to identifying the most influential topological features for the synchronizability and the spreading dynamics on networks. Both phenomena have been reported to depend on the heterogeneity of node degrees, ${ }^{50,51}$ the clustering coefficient, ${ }^{52}$ the degree correlations, ${ }^{53,54}$ the betweenness centrality, ${ }^{55}$ the $k$-coredness, ${ }^{56}$ and the community organization. ${ }^{57,58}$ Our observations indicate that the mechanism by which specific features, e.g., hubs, clustering or communities, affect the collective dynamics is through their power to alter the global path structure of the network.

We have developed a graph measure, the topological similarity $\mathcal{T}$, which estimates the expected cross-correlation between nodes based on the similarity of the estimated "influences" two nodes receive from the whole network. If two nodes receive the same sets of inputs, then they will tend to be strongly correlated. In graph analysis, the similarity of nodes is typically characterized by the matching index, which is calculated as the number of common neighbors shared by two nodes. However, the matching index only accounts for the direct links and ignores the indirect paths. The topological similarity is a generalization of the matching index which accounts for the weighted influence that every node receives from all the others along all possible paths, including recurrent and redundant ones. Indeed, it has recently been shown that the presence of ensembles of alternative paths seems to increase the overall network resiliency. ${ }^{39}$

When introducing $\mathcal{T}$, we have stated that the topological similarity can be regarded as the expected cross-correlation matrix emerging due to the underlying network structure. In fact, it was recently shown that considering communicability as the propagator kernel for a linear system of noise diffusion, the time-average cross-correlation matrix $R$ of the system could be analytically approximated. ${ }^{43}$ We have found that both approaches, the one starting from a dynamical system describing the propagation of perturbations and the topological one presented here, are indeed equivalent. The equivalence holds when all nodes are identical, i.e., have the same relaxation time-constant, and when the Gaussian noise fed into each node has the same variance and amplitude (see supplementary material).

\section{A. Structure-function relation in the human brain}

As a practical example, we have explored the contribution of the anatomical architecture to the spontaneous correlation structure measured with functional imaging. To this aim, we have calculated the topological similarity, $\mathcal{T}_{S C}$, out of the tractography-based structural connectome (SC) and have compared it to empirically obtained functional connectivity (FC) from resting-state fMRI. See also Fig. S5 (supplementary material) for a comparison between the different abilities of the communicability and the topological similarity in numerically approximating the empirical FC. The result shows that $\mathcal{T}_{S C}$ partly captures the empirical FC without fully reproducing it. This difference is to be expected, as the collective network dynamics depend do not only on the underlying anatomical connectivity but also on the state-dependent dynamical regimes of brain regions (e.g., awake state, sleep, or anesthesia) and the manner in which information is passed from one to another. By definition, topological similarity does not account for those dynamical factors; it captures the propensity of nodes to correlate or synchronize, thanks to the topology of the network they are embedded into. This tendency will then be modulated by the particular local dynamics and the coupling function of the system at hand.

Recently, a similar formalism as the one we used here has been applied but intended to find an optimal fit between the structural and the functional connectivities. ${ }^{59,60}$ The underlying assumption in these works is that the empirical FC matrix $F$ can be directly estimated from the weighted adjacency matrix $A$ of the $\mathrm{SC}$, such that there exists a function $f$ which transforms $F=f(A)$. Given $f$ to be the power series $\sum_{l=0}^{\infty} \beta_{l} A^{l}$, the goal is thus to estimate the coefficients $\left\{\beta_{l}\right\}$ leading to a best fit between $F$ and the empirical FC. It shall be noted, however, that the optimal coefficients found in such cases are difficult to interpret from a physical perspective. Because $F$ also depends on the local dynamics and the coupling function (let's denote them as $M$ and $H$ respectively), the actual estimation problem should be defined as $F=f(A, M, H)$. By assuming that $F$ depends only on $A$ such that $F=f(A)$, the optimal coefficients intrinsically carry information about the other hidden variables $M$ and $H$ which were ignored from the optimization problem. It shall be emphasized that the rationale behind $\mathcal{T}$ is not that of optimizing $F$ as a function of $A$ but instead it is that of providing a theoretical explanation for the mechanism through which the structure of a network shapes the propensity of nodes to correlate with each other.

Additionally, we have simulated the resting brain activity by modeling the local node dynamics as nonlinear units with external noise. The FC resulting from the simulations improves the accuracy to approximate the empirical FC beyond the structural contribution described by $\mathcal{T}_{\mathcal{S C}}$. However, it does not fully reproduce the empirical observation. Our results are in accordance with previous models of resting-state brain activity whose ability to replicate the empirical FC has been systematically reviewed in Refs. 10 and 27. The whole-brain network models proposed so far could only capture the empirical FC to a limited degree, with the closest models exhibiting a correlation of $r \approx 0.6$ between simulated and empirical FC. These results indicate that many of the factors determining the generation of spontaneous brain fluctuations still elude our ability to capture them. Whether these limitations are due to the local model selection or other reason, e.g., the precision of tractography to identify fiber tracts, remains a question for further investigation, although the existence of different state-dependent correlation patterns (as seen in rest, sleep, or anesthesia) sustained by the same underlying network clearly demonstrate the crucial role of dynamical factors in determining the emergent collective activity. Accordingly, interpretation of the topological similarity here introduced as a model to reproduce the empirical FC (either at rest, sleep, or under anesthesia) shall be avoided, since its role is that of exposing the expected patterns of correlation due to the anatomical structure alone, patterns which are known to be altered by the global brain state. 


\section{B. Limitations}

The results presented in this paper come with some limitations which shall be emphasized. (i) Topological similarity can be regarded as a topological estimation of the expected time-averaged cross-correlation. Thus, it is by definition blind to transient temporal fluctuations in the correlation patterns which may emerge in the network. (ii) We have here based the calculation of $\mathcal{T}$ on the communicability measure $^{41}$ which assumes an arbitrary decay of the influence with the length of the path. Although the precise decay rate of the signals may differ across real systems, our choice of the communicability guarantees that the accumulation of influence, Eq. (2), converges for all adjacency matrices $A$. In principle, the concept of topological similarity is independent of the definition of communicability; any other decay of the influence along the path can be used to estimate $\mathcal{T}$, as long as the chosen set of coefficients $\left\{\beta_{l}\right\}$ ensure the convergence of the power-series for the adjacency matrix and monotonically decays with $l$. (iii) For the analysis on the brain's connectivity and spontaneous correlations, we considered both hemispheres as if they were independent. The motivation was to avoid biases due to the unreliability of tractography to identify inter-hemispheric fibers. Still, our comparisons are biased to some extent because the SC-based $\mathcal{T}$ and simulations consider both hemispheres as independent while the empirical resting-state measurements reflect the activity of the brain regions, which are certainly embedded on the whole network.

\section{MATERIALS AND METHODS}

Twenty-one healthy volunteers (mean age 21.56 years; standard deviation 1.84 years; all males; all right handed) participated in five (5) resting-state and two (2) DTI scanning sessions and signed an informed consent. The study was conducted in the School of Psychology, Birmingham and was approved by the University of Birmingham Ethics Committee.

\section{A. Data acquisition}

Scanning sessions were conducted at the Birmingham University Imaging Centre using a $3 \mathrm{~T}$ Philips Achieva MRI scanner with a 32-channel head coil. T1-weighted anatomical data (175 slices; $1 \times 1 \times 1 \mathrm{~mm}^{3}$ resolution) were collected during the first scanning session and DTI data were collected in two sessions (23.3 \pm 2.5 days apart). The DTI acquisition consisted of 60 isotropically distributed diffusion weighted directions $\left(b=1500 \mathrm{smm}^{-2}\right.$; $\mathrm{TR}=9.5 \mathrm{~s} ; \mathrm{TE}=78 \mathrm{~ms} ; 75$ slices; $2 \times 2 \times 2 \mathrm{~mm}^{3}$ resolution; SENSE) plus a single volume without diffusion weighting $\left(b=0 \mathrm{smm}^{-2}\right.$, denoted as $\left.\mathrm{b} 0\right)$. The DTI sequence was repeated twice during each session, once following the Anterior-to-Posterior phase-encoding direction and once the Posterior-to-Anterior direction, to correct for susceptibility-induced geometric distortions. ${ }^{61}$ Resting-state data were collected in five sessions (the first and the last collected in the same scanning session as the DTI data) using whole brain echo-planar imaging (EPI) (180 volumes; $\mathrm{TR}=2 \mathrm{~s} ; \mathrm{TE}=35 \mathrm{~ms} ; 32$ slices; $2.5 \times 2.5 \times 4 \mathrm{~mm}^{3}$ resolution). Participants were instructed to have their eyes open and maintain fixation to a white dot presented at the centre of the screen.

\section{B. Whole-brain DTI tractography}

We processed the DTI data in FSL version 5.0.8 (FMRIB Software Library, http://fsl.fmrib.ox.ac.uk/fsl/ fslwiki/). We first corrected the data for susceptibility distortions, eddy currents, and motion artifacts ${ }^{62}$ and rotated the gradient directions (bvecs) to correct them for motion rotation. ${ }^{44,63,64}$ We then generated a distribution model in each voxel using FSL Bedpost $X^{65}$ with default parameters.

We parcellated the brain into 116 areas using the Automated Anatomical Labeling (AAL) atlas. ${ }^{66} \mathrm{We}$ followed a 4-step registration procedure to align the AAL atlas from Montreal Neurological Institute (MNI) template to native space: (a) align the non-weighted diffusion volume (b0) of each session to their midspace and create a midspacetemplate (rigid-body), ${ }^{67,68}$ (b) align the midspace-template to the anatomical (T1) scan (rigid-body), (c) align the T1 to the MNI template of FSL (non-linear), and (d) invert and combine all the transformation matrices of the previous steps to obtain the MNI-to-native registration. The final matrix was applied to the AAL atlas (nearest-neighbour interpolation was used in order to preserve discrete labeling values). The results of each step were visually inspected to ensure that the alignment was successful.

We simulated tracts (i.e., probabilistic streamlines) starting from each $\mathrm{AAL}$ area and reaching any other AAL area using the Probabilistic Tracking algorithm (ProbtrackX) ${ }^{69}$ The parameters we used in ProbtrackX are: 5000 samples per voxel, 2000 steps per sample until conversion, $0.5 \mathrm{~mm}$ step length, 0.2 curvature threshold, 0.01 volume fraction threshold and loopcheck enabled to prevent streamlines from forming loops. We normalised the tracts by the size of the seed area and thresholded the normalised tracts at $1 \%$ of the maximum value (i.e., setting them to zero). We subsequently computed the undirected structural connectivity matrix by averaging the normalised tracts from area $i$ to area $j$ and from area $j$ to area $i$, as directionality of the reconstructed fiber tracts cannot be inferred from DTI.

\section{Population-average structural connectome}

To estimate the population average structural connectivity (SC), we pooled the 42 SC matrices together (2 per subject) and considered both the complete parcellation having cortical, subcortical, and cerebellar regions of interest (ROIs), and the reduced one (90 brain areas - 45 per hemisphere-, which excluded the 26 regions of the cerebellum and the vermis). The $42 \mathrm{SC}$ matrices for both parcellation contained a variable number $L$ of undirected links ranging from $L=895$ for the sparsest case (density $\rho=0.22$ ) to $L=1279$ for the densest $(\rho=0.32)$. We noticed that the simple average of the matrices into a single SC matrix by averaging the 42 values, each link taken along the pool leads to an average connectome with strongly biased network properties. For example, this plain average SC matrix contained $L=1967$ links, which are almost twice the number of links as in the individual matrices. 
In order to avoid this problem, we have devised a method which automatically removes the outlier links before performing the average. For each link $(i, j)$, we have initially a set of 42 weights $\left\{w_{i j}^{s}\right\}$ where $s=1,2, \ldots, 42$. The method searches for outlier weights (data-points falling out of 1.5 times the inter-quartile range) and removes them from the data pool. The search is iteratively repeated until no further outliers are detected and then the population-average SC weight for the link $(i, j)$ is calculated as the average weight of the surviving values. In practice, the method converges very rapidly, and it rarely performs more than 2 iterations per link. This method allows us to clean the data without having to set an arbitrary hard threshold ${ }^{70}$ for the minimally accepted prevalence of the link. Full details of the method are currently in preparation and will be presented somewhere else. The resulting population-average SC matrix out of our iterative pruning method contains $L=1189$ links ( $\rho=0.30$ ), which lies within the range of connectivity for the individual 42 matrices.

\section{Resting-state time-courses and functional connectome}

We pre-processed the EPI resting-state data in FSL version 5.0.8 (FMRIB Software Library, http://fsl.fmrib.ox.ac.uk/ fsl/fslwiki/) using MELODIC (Multivariate Exploratory Linear Optimized Decomposition into Independent Components). We corrected the data for motion and slice scan timing, removed the non-brain tissue, applied $5 \mathrm{~mm}$ FWHM spatial smoothing and removed spike motion artifacts using WaveletDespike. ${ }^{71}$ We subsequently applied high-pass temporal filtering and then extracted the average timecourse from each AAL area. To estimate the population-average functional connectivity (FC) matrix, we concatenated the 105 sequences of resting-state signals ( 21 subjects, 5 sessions per subject) into a single long multivariate time-series and computed the Pearson correlation for every pair of signals. The opposite procedure, to compute an FC matrix per session and averaging over the 105 FC matrices, leads to almost identical results.

\section{E. Hopf normal model}

Within this model, the temporal evolution of the activity $z$ of node $j$ is given in the complex domain as

$$
\begin{gathered}
\frac{d z_{j}}{d t}=\left[\alpha_{j}+i \omega_{j}-\left|z^{2}\right|\right]+\sigma \eta_{j}(t), \\
z_{j}=\rho_{j} e^{i \theta_{j}}=x_{j}+i y_{j},
\end{gathered}
$$

where $\omega$ is the node's intrinsic frequency of oscillation, $\alpha$ is the local bifurcation parameter (local because the model allows the possibility to assign a different value of $\alpha$ for each node in the network), and $\eta$ is the additive Gaussian noise with standard deviation $\sigma$. This system has a supercritical bifurcation at $\alpha=0$. If $\alpha_{j}<0$, then the local dynamic has a stable fixed point at $z_{j}=0$, while for $\alpha_{j}>0$, the nodes follow a stable limit-cycle oscillation of frequency $f=\omega / 2 \pi$. Whole-brain dynamics are described by the following coupled equations:

$$
\begin{aligned}
& \frac{d x_{j}}{d t}=\left[\alpha_{j}-x_{j}^{2}-y_{j}^{2}\right] x_{j}-\omega_{j} y_{j}+g \sum_{i=1}^{N} C_{i j}\left(x_{i}-x_{j}\right)+\sigma \eta_{x j}(t), \\
& \frac{d y_{j}}{d t}=\left[\alpha_{j}-x_{j}^{2}-y_{j}^{2}\right] y_{j}+\omega_{j} x_{j}+g \sum_{i=1}^{N} C_{i j}\left(y_{i}-y_{j}\right)+\sigma \eta_{y j}(t),
\end{aligned}
$$

where $C_{i j}$ is the anatomical connectivity between nodes $i$ and $j, g$ is the global coupling factor, and the standard deviation of gaussian noise is $\sigma=0.02$. In this model, the simulated activity corresponds to the BOLD signal of each node. The intrinsic frequency of each node was estimated as the peak frequency in the associated narrowband (i.e., $0.04-0.07 \mathrm{~Hz}$ (Ref. 72)) of the empirical BOLD signals of each brain region. We simulated, for each of the two hemispheres (45 ROIs each), 330000 points using Euler's method for integration $(\mathrm{dt}=0.001)$. The connectivity between all the regions of interest was defined using the empirical structural connectivity matrix (SC) and obtained time-series were then used to compute the simulated correlation matrix (Simulated FC) by Pearson cross-correlations of the resulting time series.

\section{SUPPLEMENTARY MATERIAL}

See supplementary materials for the formal demonstration of the equivalence between $\mathcal{T}$ and $R$ when all network nodes are assumed to be identical, and they all receive a Gaussian white noise of the same intensity and variance, together with five supplementary figures: the first supplementary figure illustrates the effect of direct single link's weight on both the communicability and the topological similarity between the two nodes; the second supplementary figure shows the quantitative effect of varying links' weights in chain and cyclic topologies; the third supplementary figure depicts the behavior of four different measures of model fitting, namely Euclidean distance, mean squared error, mean absolute error, and Pearson's correlation coefficient; the fourth supplementary figure demonstrates the capacity of $\mathcal{T}$ to capture also the influence of indirect paths on the correlation observed between brain regions that, according to the empirical SC matrix, are not directly connected; the last supplementary figures show the difference between communicability and topological similarity in numerically approximating the empirical correlation structure.

\section{ACKNOWLEDGMENTS}

We would like to thank Rui Wang and Caroline di Bernardi Luft for their help in collecting the data used in this study. This work was supported by (R.G.B.) the FI-DGR scholarship of the Catalan Government through the Agència de Gestió d'Ajuts Universitari i de Recerca, under Agreement No. 2013FI-B1-00099, (G.Z.L.) the European Union's Horizon 2020 research and innovation programme under Grant Agreement No. 720270 (HBP SGA1), (G.D.) the European Research Council Advanced Grant: DYSTRUCTURE (295129) and the Spanish Research Project No. PSI201342091-P, (Z.K.) European Community's Seventh Framework 
Programme [FP7/2007-2013] under agreement PITN-GA2011-290011, (V.M.K.) European Community's Seventh Framework Programme [FP7/2007-2013] under Agreement No. PITN-GA-2012-316746 and (M.L.K.) by the European Research Council Consolidator Grant No. CAREGIVING (615539).

${ }^{1}$ D. Baker and A. Sali, "Protein structure prediction and structural genomics," Science 294, 93-96 (2001).

${ }^{2}$ D. Lee, O. Redfern, and C. Orengo, "Predicting protein function from sequence and structure," Nat. Rev. Mol. Cell Biol. 8, 995-1005 (2007).

${ }^{3}$ A. Arenas, A. Díaz-Guilera, J. Kurths, Y. Moreno, and C. S. Zhou, "Synchronization in complex networks," Phys. Rep. 469, 93-153 (2008).

${ }^{4}$ S. Boccaletti, V. Latora, Y. Moreno, M. Chavez, and D.-U. Hwang, "Complex networks: Structure and dynamics," Phys. Rep. 424, 175-308 (2006).

${ }^{5}$ S. Pei and H. A. Makse, "Spreading dynamics in complex networks," J. Stat. Mech. 12, P12002 (2013).

${ }^{6}$ R. Pastor-Satorras, C. Castellano, P. van Mieghem, and A. Vespignani, "Epidemic processes in complex networks," Rev. Mod. Phys. 87, 925-979 (2015).

${ }^{7}$ L. M. Pecora and T. L. Carroll, "Master stability functions for synchronized coupled systems," Phys. Rev. Lett. 80, 2109-2112 (1998).

${ }^{8}$ L. Huang, Q. Chen, Y.-C. Lai, and L. Pecora, "Generic behaviour of master-stability functions in coupled nonlinear dynamical systems," Phys. Rev. E 80, 036204 (2009).

${ }^{9}$ G. Schmidt, G. Zamora-López, C. Zhou, and J. Kurths, "Simulation of large scale cortical networks by individual neuron dynamics," Int. J. Bifurcation Chaos 20, 859-867 (2010).

${ }^{10}$ A. Messé, D. Rudrauf, H. Benali, and G. Marrelec, "Relating structure and function in the human brain: Relative contributions of anatomy, stationary dynamics, and non-stationarities," PLoS Comput. Biol. 10, e1003530 (2014).

${ }^{11}$ J. S. Damoiseaux and M. D. Greicius, "Greater than the sum of its parts: a review of studies combining structural connectivity and resting-state functional connectivity," Brain Struct. Funct. 213, 525-533 (2009).

${ }^{12}$ D. J. Sharp, C. F. Beckmann, R. Greenwood, K. M. Kinnunen, V. Bonnelle, X. De Boissezon, J. H. Powell, S. J. Counsell, M. C. Patel, and R. Leech, "Default mode network functional and structural connectivity after traumatic brain injury," Brain 134, 2233-2247 (2011).

${ }^{13}$ X. Shen, E. S. Finn, D. Scheinost, M. D. Rosenberg, M. M. Chun, X. Papademetris, and R. T. Constable, "Using connectome-based predictive modeling to predict individual behavior from brain connectivity," Nat. Protocols 12, 506-518 (2017).

${ }^{14}$ B. Biswal, F. Zerrin Yetkin, V. M. Haughton, and J. S. Hyde, "Functional connectivity in the motor cortex of resting human brain using echo-planar MRI," Magn. Reson. Med. 34, 537-541 (1995).

${ }^{15}$ D. A. Gusnard and M. E. Raichle, "Searching for a baseline: Functional imaging and the resting human brain," Nat. Rev. Neurosci. 2, 685-694 (2001).

${ }^{16}$ M. D. Fox, A. Z. Snyder, J. L. Vincent, M. Corbetta, D. C. V. Essen, and M. E. Raichle, "The human brain is intrinsically organized into dynamic, anticorrelated functional networks," Proc. Natl. Acad. Sci. U.S.A. 102, 9673-9678 (2005).

${ }^{17}$ C. F. Beckmann, M. DeLuca, J. T. Devlin, and S. M. Smith, "Investigations into resting-state connectivity using independent component analysis," Philos. Trans. R. Soc. B 360, 1001-1013 (2005).

${ }^{18}$ B. B. Biswal, M. Mennes, X.-N. Zuo, S. Gohel, C. Kelly, S. M. Smith, C. F. Beckmann, J. S. Adelstein, R. L. Buckner, S. Colcombe, A.-M. Dogonowski, M. Ernst, D. Fair, M. Hampson, M. J. Hoptman, J. S. Hyde, V. J. Kiviniemi, R. Kötter, S.-J. Li, C.-P. Lin, M. J. Lowe, C. Mackay, D. J. Madden, K. H. Madsen, D. S. Margulies, H. S. Mayberg, K. McMahon, C. S. Monk, S. H. Mostofsky, B. J. Nagel, J. J. Pekar, S. J. Peltier, S. E. Petersen, V. Riedl, S. A. R. B. Rombouts, B. Rypma, B. L. Schlaggar, S. Schmidt, R. D. Seidler, G. J. Siegle, C. Sorg, G.-J. Teng, J. Veijola, A. Villringer, M. Walter, L. Wang, X.-C. Weng, S. Whitfield-Gabrieli, P. Williamson, C. Windischberger, Y.-F. Zang, H.-Y. Zhang, F. X. Castellanos, and M. P. Milham, "Toward discovery science of human brain function," Proc. Natl. Acad. Sci. U.S.A. 107, 4734-4739 (2010).

${ }^{19}$ C. S. Zhou, L. Zemanová, G. Zamora-López, C.-C. Hilgetag, and J. Kurths, "Hierarchical organization unveiled by functional connectivity in complex brain networks," Phys. Rev. Lett. 97, 238103 (2006).
${ }^{20}$ C. J. Honey, R. Kotter, M. Breakspear, and O. Sporns, "Network structure of cerebral cortex shapes functional connectivity on multiple time scales," Proc. Natl. Acad. Sci. U.S.A. 104, 10240-10245 (2007).

${ }^{21}$ J. Gómez-Gardeñes, G. Zamora-López, Y. Moreno, and A. Arenas, "From modular to centralized organization of synchronization in functional areas of the cat cerebral cortex," PLoS One 5, e12313 (2010).

${ }^{22}$ G. Deco, V. Jirsa, A. R. McIntosh, O. Sporns, and R. Kotter, "Key role of coupling, delay, and noise in resting brain fluctuations," Proc. Natl. Acad. Sci. U.S.A. 106, 10302-10307 (2009).

${ }^{23}$ G. Deco, V. K. Jirsa, and A. R. McIntosh, "Emerging concepts for the dynamical organization of resting-state activity in the brain," Nat. Rev. Neurosci. 12, 43-56 (2011).

${ }^{24}$ F. Abdelnour, H. U. Voss, and A. Raj, "Network diffusion accurately models the relationship between structural and functional brain connectivity networks," NeuroImage 90, 335-347 (2014).

${ }^{25}$ A. Messé, H. Benali, and G. Marrelec, "Relating structural and functional connectivity in MRI: A simple model for a complex brain," IEEE Trans. Med. Imaging 34, 27-37 (2015).

${ }^{26}$ M. L. Saggio, P. Ritter, and V. K. Jirsa, "Analytical operations relate structural and functional connectivity in the brain," PloS One 11, e0157292 (2016).

${ }^{27}$ A. Messé, D. Rudrauf, A. Giron, and G. Marrelec, "Predicting functional connectivity from structural connectivity via computational models using MRI: An extensive comparison study," NeuroImage 111, 65-75 (2015).

${ }^{28}$ G. Deco, P. Hagmann, A. Hudetz, and G. Tononi, "Modeling resting-state functional networks when the cortex falls asleep: Local and global changes," Cereb. Cortex 24, 3180-3194 (2014).

${ }^{29}$ A. Hudetz, X. Liu, and S. Pillay, "Dynamic repertoire of intrinsic brain states is reduced in propofol-induced unconsciousness," Brain Connect. 5, $1-13$ (2014).

${ }^{30}$ R. G. Bettinardi, N. Tort-Colet, M. Ruiz-Mejias, M. V. Sanchez-Vives, and G. Deco, "Gradual emergence of spontaneous correlated brain activity during fading of general anesthesia in rats: Evidences from fMRI and local field potentials," NeuroImage 114, 185-198 (2015).

${ }^{31}$ V. Latora and M. Marchiori, "Efficient behavior of small-world networks," Phys. Rev. Lett. 87, 198701 (2001).

${ }^{32}$ B. A. Huberman and L. A. Adamic, "Information dynamics in the networked world," in Complex Networks, Lecture Notes in Physics Vol. 650, edited by E. Ben-Naim, H. Frauenfelder, and Z. Toroczkai (Springer, Berlin, Heidelberg, 2004), pp. 371-398.

${ }^{33}$ D. J. Ashton, T. C. Jarrett, and N. F. Johnson, "Effect of congestion costs on shortest paths through complex networks," Phys. Rev. Lett. 94, 058701 (2005).

${ }^{34}$ A. Trusina, M. Rosvall, and K. Sneppen, "Communication boundaries in networks," Phys. Rev. Lett. 94, 238701 (2005).

${ }^{35}$ S. P. Borgatti, "Centrality and network flow," Soc. Networks 27, 55-71 (2005).

${ }^{36}$ V. Colizza, A. Flammini, M. A. Serrano, and A. Vespignani, "Detecting rich-club ordering in complex networks," Nat. Phys. 2, 110-115 (2006).

${ }^{37}$ G. Zamora-López, C. S. Zhou, and J. Kurths, "Graph analysis of cortical networks reveals complex anatomical communication substrate," Chaos 19, 015117 (2009).

${ }^{38}$ J. Goñi, M. P. v. d. Heuvel, A. Avena-Koenigsberger, N. V. d. Mendizabal, R. F. Betzel, A. Griffa, P. Hagmann, B. Corominas-Murtra, J.-P. Thiran, and O. Sporns, "Resting-brain functional connectivity predicted by analytic measures of network communication," Proc. Natl. Acad. Sci. U.S.A. 111, 833-838 (2014).

${ }^{39}$ A. Avena-Koenigsberger, B. Mišić, R. Hawkins, A. Griffa, P. Hagmann, J. Goñi, and O. Sporns, "Path ensembles and a tradeoff between communication efficiency and resilience in the human connectome," Brain Struct. Funct. 222(1), 603-618 (2016).

${ }^{40}$ J. Bang-Jensen and G. Z. Gutin, Digraphs: Theory, Algorithms and Applications (Springer, 2008)

${ }^{41}$ E. Estrada and N. Hatano, "Communicability in complex networks," Phys. Rev. E 77, 036111 (2008).

${ }^{42}$ E. Estrada, N. Hatano, and M. Benzi, "The physics of communicability in complex networks," Phys. Rep. 514, 89-119 (2012).

${ }^{43}$ G. Zamora-López, Y. Chen, G. Deco, M. L. Kringelbach, and C. Zhou, "Functional complexity emerging from anatomical constraints in the brain: the significance of network modularity and rich-clubs," Sci. Rep. 6, 38424 (2016).

${ }^{44} \mathrm{D}$. K. Jones and M. Cercignani, "Twenty-five pitfalls in the analysis of diffusion MRI data," NMR Biomed. 23, 803-820 (2010). 
${ }^{45}$ C. Thomas, Q. Y. Frank, M. O. Irfanoglu, P. Modi, K. S. Saleem, D. A. Leopold, and C. Pierpaoli, "Anatomical accuracy of brain connections derived from diffusion MRI tractography is inherently limited," Proc. Natl. Acad. Sci. U.S.A. 111, 16574-16579 (2014).

${ }^{46}$ S. Jbabdi, S. N. Sotiropoulos, S. N. Haber, D. C. Van Essen, and T. E. Behrens, "Measuring macroscopic brain connections in vivo," Nat. Neurosci. 18, 1546-1555 (2015).

${ }^{47}$ F. Freyer, J. A. Roberts, R. Becker, P. A. Robinson, P. Ritter, and M. Breakspear, "Biophysical mechanisms of multistability in resting-state cortical rhythms," J. Neurosci. 31, 6353-6361 (2011).

${ }^{48}$ F. Freyer, J. A. Roberts, P. Ritter, and M. Breakspear, "A canonical model of multistability and scale-invariance in biological systems," PLoS Comput Biol. 8, e1002634 (2012).

${ }^{49}$ G. Deco and M. L. Kringelbach, "Metastability and coherence: Extending the communication through coherence hypothesis using a whole-brain computational perspective," Trends Neurosci. 39, 125-135 (2016).

${ }^{50}$ Y. Moreno, R. Pastor-Satorras, and A. Vespignani, "Epidemic outbreaks in complex heterogeneous networks,” Eur. Phys. J. B 26, 521-529 (2002).

${ }^{51} \mathrm{C}$. Zhou and J. Kurths, "Hierarchical synchronization in networks of oscillators with heterogeneous degrees," Chaos 16, 015104 (2006).

${ }^{52} \mathrm{X}$. Wu, B. Wang, T. Zhou, W. Wang, M. Zhao, and H. Yang, "Synchronizability of highly clustered scale-free networks," Chin. Phys. Lett. 23(4), 1046-1049 (2006).

${ }^{53}$ M. di Bernardo, F. Garofalo, and F. Sorrentino, "Effects of degree correlation on the synchronizability of networks of nonlinear oscillators," in Proceedings of the 44th IEEE Conference on Decision and Control, and the European Control Conference WeA14.1 (2005).

${ }^{54}$ M. Nekovee, Y. Moreno, G. Bianconi, and M. Marsili, "Theory of rumour spreading in complex social networks," Physica A 374, 457-470 (2007).

${ }^{55}$ M. Chavez, D.-U. Hwang, A. Amann, and S. Boccaletti, "Synchronizing weighted complex networks," Chaos 16, 015106 (2006).

${ }^{56}$ M. Kitsak, L. K. Gallos, S. Havlin, F. Liljiero, L. Muchnik, H. E. Stanley, and H. A. Makse, "Identification of influential spreaders in complex networks,” Nat. Phys. 6, 888-893 (2010).

${ }^{57}$ A. Arenas, A. Díaz-Guilera, and C. Pérez-Vicente, "Synchronization reveals topological scales in complex networks," Phys. Rev. Lett. 96, 114102 (2006).

${ }^{58}$ R. A. Baños, J. Borge-Holthoefer, and Y. Moreno, "The role of hidden influentials in the diffusion of online information cascades," Eur. Phys. J. B 2, 6 (2013).

${ }^{59}$ J. Meier, P. Tewarie, L. Douw, B. W. van Dijk, S. M. Stufflebeam, and P. van Mieghem, "A mapping between structural and functional brain networks," Brain Connect. 6, 298-311 (2016).
${ }^{60} \mathrm{H}$. Liang and H. Wang, "Structure-function network mapping and its assessment via persistent homology," PLoS Comput. Biol. 13, e1005325 (2017).

${ }^{61}$ J. L. Anderson, S. Skare, and J. Ashburner, "How to correct susceptibility distortions in spin echo-planar images: Application to diffusion tensor imaging," NeuroImage 20, 870-888 (2003).

${ }^{62}$ J. Anderson and S. Sotiropoulos, "An integrated approach to correction for off-resonance effects and subject movement in diffusion MR imaging," NeuroImage 125, 1063-1078 (2016).

${ }^{63} \mathrm{~A}$. Leemans and D. Jones, "The B-matrix must be rotated when correcting for subject motion in DTI data," Magn. Reson. Med. 61, 1336 (2009).

${ }^{64}$ A. Ersiz, V. E. Arpinar, S. Dreyer, and L. T. Muftuler, "Quantitative analysis of the efficacy of gradient table correction on improving the accuracy of fiber tractography," Magn. Reson. Med. 72, 227-236 (2014).

${ }^{65}$ T. E. Behrens, H. J. Berg, S. Jbabdi, M. F. S. Rushworth, and M. W. Woolrich, "Probabilistic diffusion tractography with multiple fibre orientations: What can we gain?" NeuroImage 34(1), 144-155 (2003).

${ }^{66}$ N. Tzourio-Mazoyer, B. Landeau, D. Papathanassiou, F. Crivello, O. Etard, N. Delcroix, B. Mazoyer, and M. Joliot, "Automated anatomical labeling of activations in SPM using a macroscopic anatomical parcellation of the MNI MRI single-subject brain," Neuroimage 15, 273 (2002).

${ }^{67}$ S. M. Smith, N. De Stefano, M. Jenkinson, and P. M. Matthews, "Normalized accurate measurement of longitudinal brain change," J. Comput. Assist. Tomogr. 25, 466-475 (2001).

${ }^{68} \mathrm{C}$. Thomas and C. I. Baker, "Teaching an adult brain new tricks: A critical review of evidence for training-dependent structural plasticity in humans," NeuroImage 73, 225-236 (2013).

${ }^{69}$ T. Behrens, H. Berg, S. Jbabdi, M. Rushworth, and M. Woolrich, "Probabilistic diffusion tractography with multiple fibre orientations: What can we gain?," Neuroimage 34, 144 (2007).

${ }^{70}$ M. A. de Reus and M. van den Heuvel, "Estimating false positives and negatives in brain networks," NeuroImage 70, 402-409 (2013).

${ }^{71}$ A. X. Patel, P. Kundu, M. Rubinov, P. S. Jones, P. E. Vérts, K. D. Ersche, J. Suckling, and E. T. Bullmore, "A wavelet method for modeling and despiking motion artifacts from resting-state fMRI time series," NeuroImage 95, 287-304 (2014).

${ }^{72}$ E. Glerean, J. Salmi, J. M. Lahnakoski, I. P. Jääskeläinen, and M. Sams, "Functional magnetic resonance imaging phase synchronization as a measure of dynamic functional connectivity," Brain Connect. 2, 91-101 (2012). 